\title{
Diseño de un controlador de carga de tres etapas para sistemas fotovoltaicos usando lógica difusa
}

\author{
Design of a three-states charge controller for photovoltaic systems using fuzzy logic \\ Daniel Pacheco Bautista ${ }^{1 *} \quad$ Elsa Cortés Rito $^{1} \quad$ Francisco Aguilar Acevedo $^{1}$ \\ Recibido 15 de octubre de 2017, aceptado 13 de septiembre de 2018 \\ Received: October 15, 2017 Accepted: September 13, 2018
}

\begin{abstract}
RESUMEN
En este artículo se presenta el diseño y simulación de un controlador de carga tipo buck para acumuladores de plomo-ácido utilizados en aplicaciones fotovoltaicas, empleando técnicas de control basadas en lógica difusa. El diseño propuesto implementa completamente el algoritmo de tres etapas: bulk, absorción y flotación, lo que permite realizar la carga del acumulador de manera precisa, asegurando su integridad y durabilidad. El sistema de control está constituido por dos controladores difusos independientes, el primero regula la corriente en forma exacta en la etapa de bulk, mientras que el segundo regula el voltaje en las etapas de absorción y flotación; adicionalmente, cuenta con lógica de conmutación la cual permite la transición entre las diferentes etapas del algoritmo. El sistema fue diseñado y simulado en MATLAB, los resultados muestran la funcionalidad correcta del diseño al simular la carga de acumuladores de $12 \mathrm{~V}$ con capacidades de 60 a $120 \mathrm{Ah}$. Aun cuando en la literatura existen trabajos similares, muy pocos implementan un algoritmo con múltiples etapas y que pueda realizarse de manera directa en sistemas económicos como los microcontroladores. Este trabajo puede tomarse como referencia en el diseño de controladores de carga incluso diferentes a los basados en lógica difusa, representando una importante aportación didáctica.
\end{abstract}

Palabras clave: Controlador de carga, sistema fotovoltaico, lógica difusa, controlador difuso.

\begin{abstract}
This paper presents the design and simulation of a buck charge controller for lead-acid accumulators used in photovoltaic applications, based on fuzzy control techniques. The proposed design fully implements the three-stage algorithm: bulk, absorption, and flotation, which allows the battery to be accurately charged, ensuring its integrity and durability. The control system consists of two independent fuzzy controllers, the first regulates the current accurately in the bulk stage, while the second regulates the voltage in the absorption, and flotation stages; additionally, it has switching logic, which allows transitioning between the different stages of the algorithm. The system was designed and simulated in MATLAB; and the results show the correct functionality of the design by simulating the charge of $12 \mathrm{~V}$ accumulators with capacities from 60 to $120 \mathrm{Ah}$. Even though in the literature, there are similar works, very few use an algorithm with multiple stages completely, and that can be implemented directly in economic systems such as microcontrollers. This work can be taken as a reference in the design of charge controllers even different from those based on fuzzy logic, which represents an important didactic contribution.
\end{abstract}

Keywords: Charge controller, photovoltaic system, fuzzy logic, fuzzy controller.

1 Departamento de Ingeniería en Computación. Universidad del Istmo. Ciudad Universitaria S/N, Barrio Santa Cruz Tagolaba, Tehuantepec, Oaxaca, México, C.P. 70760.

E-mail:dpachecob@bianni.unistmo.edu.mx; elsa.cortes.rito@gmail.com; aguilar.afco@sandunga.unistmo.edu.mx

* Autor de correspondencia. E-mail: dpachecob@bianni.unistmo.edu.mx 


\section{INTRODUCCIÓN}

En la actualidad la búsqueda de nuevas formas de generación de energía eléctrica se ha intensificado notablemente, como consecuencia del crecimiento exponencial en su consumo a nivel mundial. Entre las alternativas, los sistemas fotovoltaicos son una tecnología prometedora puesto que convierten un recurso sumamente abundante en energía eléctrica. Los sistemas fotovoltaicos pueden ser autónomos, en cuyo caso es fundamental el uso de acumuladores eléctricos, o bien interconectados a la red eléctrica, donde el uso de éstos puede omitirse. Un componente fundamental del primer tipo es el controlador de carga, el cual es el responsable de transmitir la energía proveniente de los paneles solares a los acumuladores de una forma precisa, de tal manera que garanticen la carga, seguridad y durabilidad de los mismos.

Los circuitos más utilizados para el proceso de carga son los convertidores conmutados CD-CD tipo buck, boost y buck-boost [1] Para controlarlos se usan generalmente esquemas clásicos, como el Proporcional-Integral (PI), el Proporcional-Derivativo (PD) o el Proporcional-Integral-Derivativo (PID); no obstante, para obtener un desempeño aceptable se requieren modelos matemáticos complejos debido a la no linealidad del circuito. Esto ha llevado a la búsqueda de técnicas diferentes para implementar su control, siendo la lógica difusa una alternativa, proporcionando un método conveniente para la construcción de controladores no lineales a través de la utilización de información heurística [2].

Existen diversos trabajos donde se utiliza la lógica difusa para el control de convertidores DC-DC. En [3] se desarrolla un controlador difuso universal para el control de un convertidor tipo boost, los resultados de su simulación muestran como el desempeño del controlador difuso es superior al de un controlador tipo PI, en diversos puntos de operación. En [4] se presenta el desarrollo de un controlador difuso de propósito general, para los convertidores buck-boost y Sepic, comparando los resultados con un controlador estándar. El controlador difuso superó en desarrollo al estándar en sensibilidad a variaciones de parámetros y en términos de estabilidad. En [5] se diseña un controlador para convertidores DC-DC, comparando su desempeño con controladores en modo corriente en los convertidores buck-boost, boost y buck. Los resultados muestran que los controladores difusos tienen mejor respuesta transitoria y menor error en estado estable. Todos estos trabajos representan un excelente referente de la capacidad del control difuso, pero ninguno de estos simula el proceso de carga completo de un acumulador.

En este artículo se diseña un controlador de carga completo siguiendo el algoritmo de tres etapas: bulk-absorción-flotación, para el convertidor CD-CD tipo buck, basado en lógica difusa. El diseño está orientado a la carga de acumuladores de plomoácido de 12 voltios con capacidades de 60 a $120 \mathrm{Ah}$, utilizados en aplicaciones fotovoltaicas. El artículo explica detalladamente la lógica de conmutación que permite la transición entre etapas, por lo que puede tomarse como referencia en el desarrollo de otros controladores de carga, sin limitarse a los basados en lógica difusa. El sistema propuesto es diseñado y simulado en MATLAB utilizando las herramientas: Fuzzy Logic, Simscape y Simulink.

El resto del artículo se organiza de la siguiente manera: en la sección subsecuente se presentan los fundamentos del controlador de carga, enfatizando en el circuito convertidor CD-CD, y el algoritmo de carga. Posteriormente se describe el uso de lógica difusa en los sistemas de control. A continuación, se presentan el diseño del sistema propuesto y los resultados de su simulación. El artículo finaliza con las conclusiones del trabajo.

\section{CONTROLADOR DE CARGA}

El controlador de carga tiene como función principal garantizar el proceso óptimo de carga del acumulador, evitando la sobrecarga y sobredescarga del mismo con el fin de alargar su vida útil. Lo anterior puede lograrse utilizando diversos métodos denominados algoritmos de carga, siendo el algoritmo de tres etapas: bulk-absorciónflotación, uno de los más completos y utilizados en aplicaciones fotovoltaicas [6].

En el algoritmo de tres etapas (ver Figura 1), el proceso de carga comienza en la etapa de bulk, inyectando al acumulador una corriente contante tan alta como lo permitan las características del mismo, generalmente entre el $10 \%$ y el $20 \%$ de su capacidad. De esta manera, el voltaje se incrementa lentamente hasta alcanzar el voltaje de absorción (valor típico entre 14,2 y $14,8 \mathrm{~V}$ para acumuladores de $12 \mathrm{~V}$ de 
plomo-ácido), en este punto el acumulador posee aproximadamente el $80 \%$ de su carga total.

Posteriormente, en la etapa de absorción se mantiene el voltaje del acumulador constante e igual al voltaje de absorción, mientras que la corriente disminuye paulatinamente hasta llegar a un valor mínimo, generalmente entre el $1 \%$ y el $2 \%$ de la capacidad del acumulador, cuando esto ocurre el dispositivo se considera cargado.

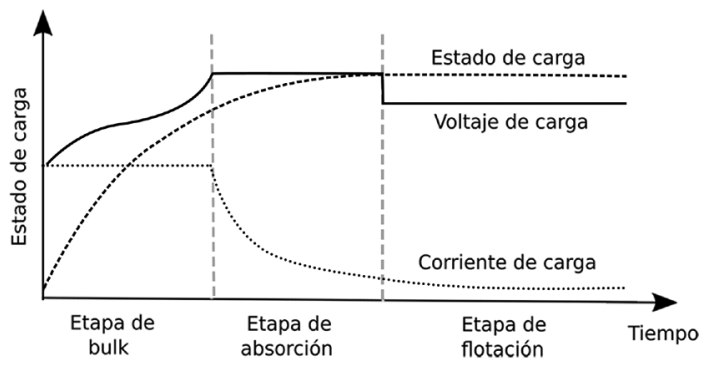

Figura 1. Algoritmo de carga de tres etapas.

Finalmente, en la etapa de flotación se mantiene una tensión constante, a un nivel más bajo del de absorción (típicamente entre 13,2 y 13,8 V) para compensar posibles descargas indeseadas del mismo. Estructuralmente el controlador de carga se compone de un convertidor CD-CD y un sistema de control. El convertidor CD-CD permite regular un voltaje continuo en otro nivel de voltaje continuo. En este trabajo se utiliza el convertidor conmutado tipo buck en su modo de operación continuo [7], el cual se muestra en la Figura 2. El transistor MOSFET opera como conmutador, de tal forma que cuando está encendido polariza inversamente al diodo, siendo la fuente de entrada quien suministra energía al inductor, al capacitor y a la carga; por el contrario, si el transistor está apagado, el diodo conduce, el capacitor alimenta energía a la carga y la corriente del inductor fluye a través del capacitor y el diodo. De esta forma el voltaje en la carga puede controlarse variando el ciclo útil de la señal en la base del transistor, esta es precisamente la función del módulo de control.

\section{CONTROL DIFUSO}

Aunque los métodos de control basados en modelos lineales están bien establecidos, estos no son muy apropiados cuando el proceso controlado es altamente no lineal como es el caso de los convertidores DC-DC [8]. Ante este panorama, el control difuso ha surgido como un importante método alternativo, especialmente en aquellos procesos que son muy complejos para analizarse por técnicas convencionales.

A diferencia del control convencional, el control difuso está basado en el conocimiento de un experto en el sistema. El control difuso provee una metodología formal para representar e implementar el conocimiento heurístico humano para controlar el sistema. Un sistema de control difuso (ver Figura 3), consta básicamente de los siguientes componentes: (1) un fusificador que convierte las entradas del sistema en información que el mecanismo de inferencia pueda utilizar para activar y aplicar reglas, (2) la base de reglas la cual contiene la descripción lingüística del experto de cómo lograr un buen control, (3) el mecanismo de inferencia que evalúa que reglas son relevantes en la situación actual y (4) el defusificador el cual convierte la conclusión del mecanismo de inferencia en entradas de control a la planta. Más adelante se detallarán los componentes cuando se realice el diseño de los controladores de voltaje y corriente.

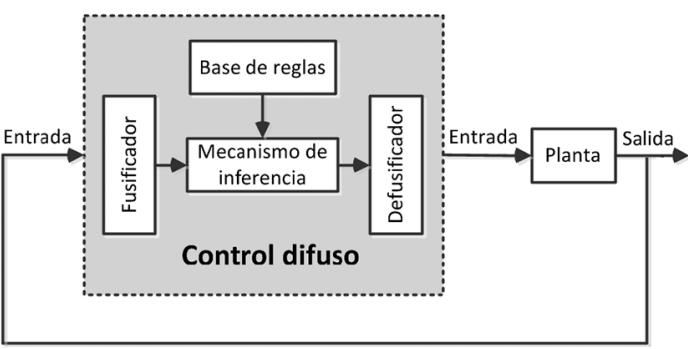

Figura 3. Diagrama a bloques del sistema de control difuso.

Figura 2. Convertidor CD-CD tipo buck. 


\section{DISEÑO DEL SISTEMA}

En la Figura 4 se muestra el diagrama a bloques del controlador de carga propuesto. El sistema está formado por dos módulos: (1) el módulo de potencia, el cual es en esencia el convertidor buck y tiene como función transferir la potencia del panel hacia el acumulador en función del ciclo de trabajo de una señal modulada en amplitud (PWM) generada en el módulo de control, y (2) el módulo de control, compuesto a su vez por dos controladores difusos uno de corriente y otro de voltaje, más lógica de conmutación. El primer controlador detecta la corriente en el acumulador y genera el voltaje de control apropiado para el módulo PWM con la finalidad de regular la corriente de carga. El segundo controlador hace una función similar, pero detectando el voltaje del acumulador con la finalidad de regular el voltaje de carga. Finalmente, la lógica de conmutación, permite cambiar entre las tres etapas del algoritmo en función del estado de carga del acumulador. A continuación, se describe a detalle el diseño de cada uno de los módulos.

\section{Módulo de potencia}

El convertidor buck fue calculado para una potencia máxima de $240 \mathrm{~W}$, voltaje de entrada de $24 \mathrm{~V}$, voltaje de salida de $14,4 \mathrm{~V}$, frecuencia de conmutación de 50 $\mathrm{KHz}$ y operación en modo continuo. Considerando un sistema alimentado por dos paneles solares

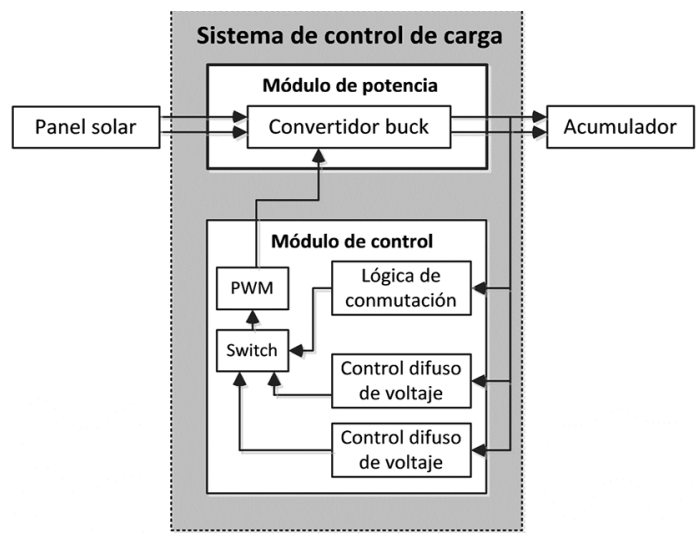

Figura 4. Diagrama a bloques del sistema propuesto.

convencionales conectados en paralelo. Obteniendo valores de $86 \mu \mathrm{H}$ y $330 \mu \mathrm{F}$ para el inductor y el capacitor respectivamente. El modelo del convertidor buck, se muestra en la Figura 5.

\section{Módulo de control}

El diseño de los controladores difusos fue desarrollado bajo las siguientes fases: (1) elección de las entradas y salidas del controlador, (2) elección del pre-procesamiento para las entradas y salidas del controlador y (3) el diseño de cada una de los cuatro bloques de la Figura 3. Ambos controladores de corriente y voltaje fueron diseñados utilizando el modelo genérico mostrado en la Figura 6. La

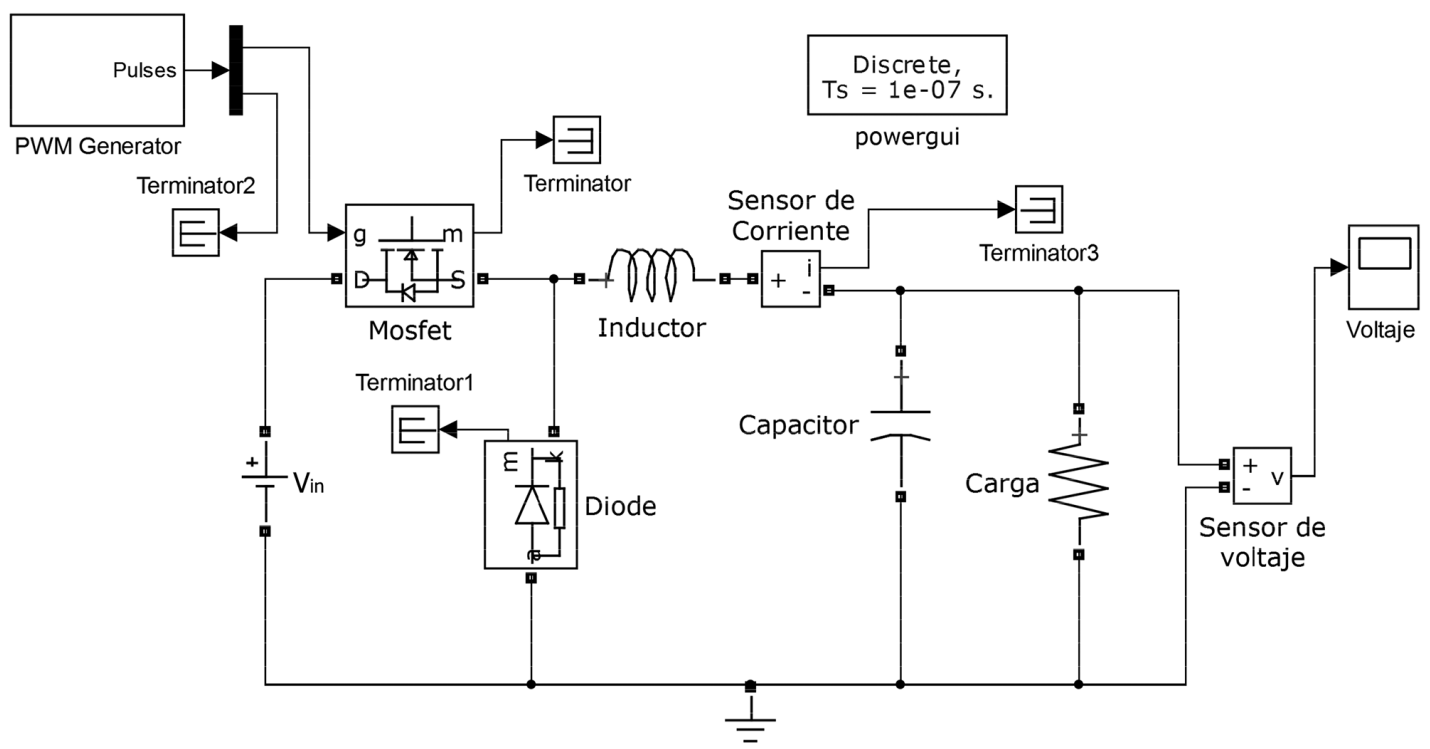

Figura 5. Modelo del convertidor buck. 
primera entrada es el error en el voltaje de salida (o corriente) $e[k]=R e f$-Acu $[k]$ donde $A c u[k]$ es el valor medido en el acumulador en la $k^{\text {th }}$ muestra y Ref es el valor correspondiente al valor deseado. La segunda entrada $\Delta e[k]=e[k]-e[k-1]$, es la diferencia entre el error de la $k^{\text {th }}$ muestra y el error de la $[k-1]^{\text {th }}$ muestra. Ambas entradas son posteriormente multiplicadas por los factores ge y gde respectivamente, y luego alimentadas al controlador difuso. La salida del controlador difuso es el cambio en el ciclo útil $\Delta u[k]$, el cual es escalado posteriormente por el factor $g u$.

Finalmente, la versión escalada del controlador difuso se integra para obtener el valor del ciclo útil, el cual habilitará al convertidor buck. La integración se logra mediante la suma acumulativa de $\Delta u[k]$ en cada ciclo de muestreo, representado matemáticamente por la ecuación (1).

$$
d[k]=d[k-1]+g u * \Delta u[k]
$$

El uso de un integrador en la salida del controlador difuso disminuye notablemente el error en estado estable. No obstante, debe tenerse cuidado en la elección del valor de $g u$, un valor muy grande provoca oscilaciones en el estado estable, mientras que uno pequeño incrementa el tiempo de respuesta transitoria [9]. Los factores ge, gde y gu son utilizados para sintonizar el sistema hasta obtener una respuesta satisfactoria. En este caso particular, las ganancias para el controlador de corriente fueron $g e=1,2, g d e=1,8$ y $g u=6$, mientras que para el controlador de voltaje se establecieron en $g e=1,2$, $g d e=1,4$ y $g u=10$.

\section{Fusificación}

El controlador de carga regulará corrientes de 6 a 12 A y voltajes de 10 a $18 \mathrm{~V}$. El rango de la corriente se ha definido considerando que la corriente máxima en la etapa de bulk debe ser el $10 \%$ de la capacidad del acumulador. En cuanto al rango del voltaje se han considerado los posibles voltajes de absorción y flotación de los acumuladores de $12 \mathrm{~V}$ para aplicaciones fotovoltaicas. Lo anterior permite establecer el universo de discurso entre -12 y $12 \mathrm{~A}$ para el error de corriente y entre -18 y $18 \mathrm{~V}$ para el error de voltaje.

Cada universo de discurso fue dividido en 5 conjuntos difusos: NG (Negativo Grande), NP (Negativo Pequeño), CE (Cero), PP (Positivo Pequeño) y PG (Positivo Grande). Las funciones de membresía del error y la derivada del error para los controladores de corriente y de voltaje se muestran en las Figuras 7 a 9 .

De forma similar, considerando los posibles valores de entrada del modulador de ancho de pulso (-1 a $1)$, se determinaron las funciones de membresía

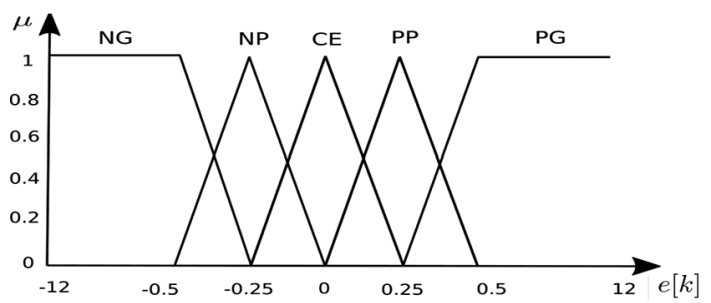

Figura 7. Funciones de membresía del Error para el controlador de corriente.

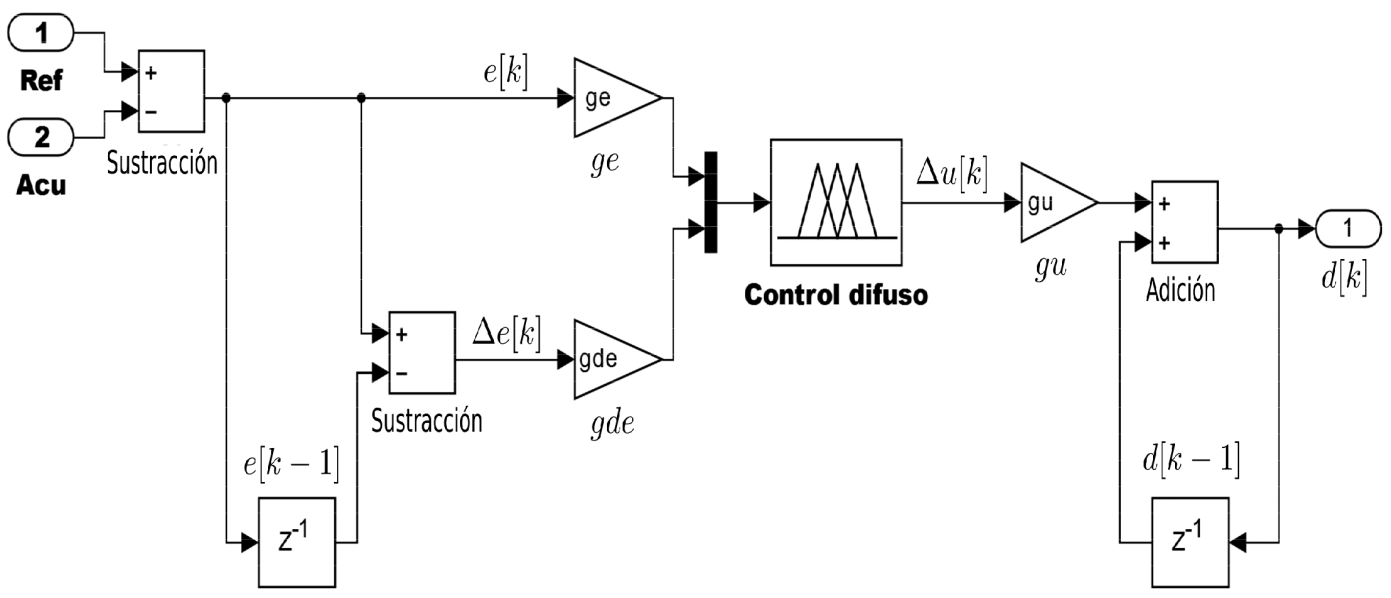

Figura 6. Modelo genérico del controlador. 


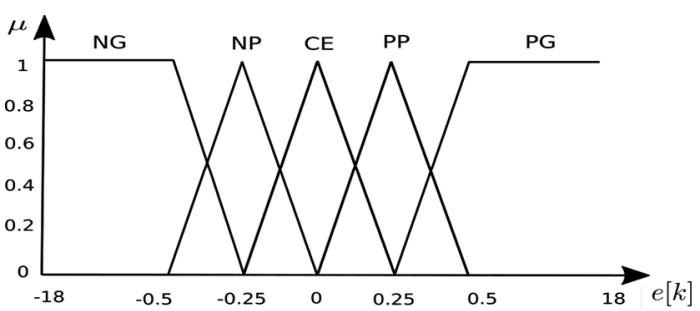

Figura 8. Funciones de membresía del Error para el controlador de voltaje.

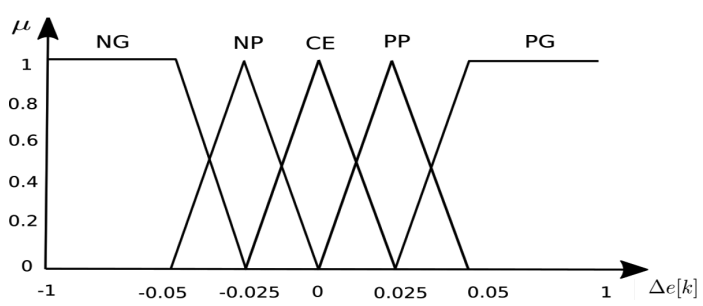

Figura 9. Funciones de membresía de la Derivada del Error para ambos controladores.

de la variable de salida $\Delta u[k]$ mostradas en las Figuras 10 y 11 .

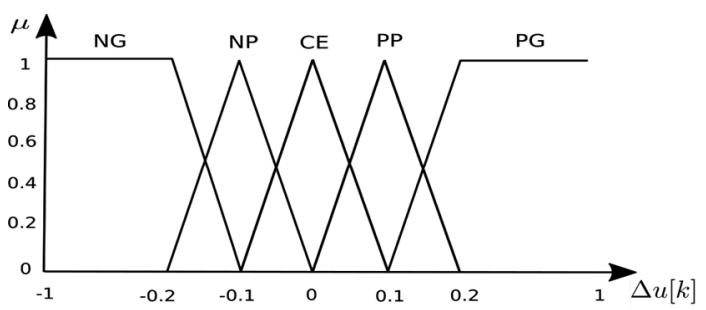

Figura 10. Funciones de membresía de Salida para el controlador de corriente.

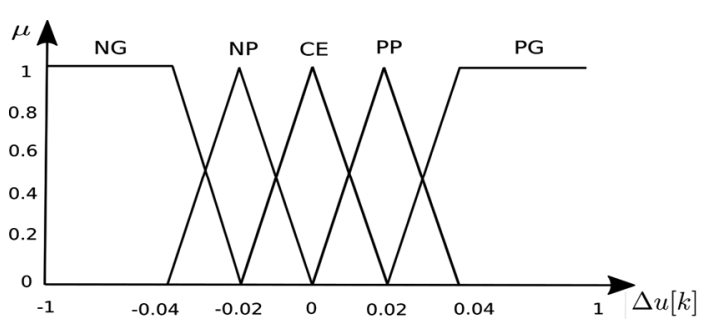

Figura 11. Funciones de membresía de Salida para el controlador de voltaje.

El número de conjuntos difusos, así como las formas de las funciones de membresía fueron elegidos para minimizar los recursos de cómputo en una implementación futura basada en microcontrolador, mientras que los intervalos de cada función de membresía fueron ajustados mediante experimentación.

\section{Base de reglas}

Las reglas difusas combinan uno o más conjuntos difusos de entrada llamados antecedentes o premisas y le asocian un conjunto difuso de salida llamado consecuente o consecuencia. Existen varios tipos de reglas, no obstante, en este trabajo se utilizó el modelo lingüístico difuso de Mamdani [10], expresando las reglas como:

\section{Si $e$ es $A$ y $\Delta$ e es $B$ entonces $\Delta u$ es $C$}

Donde $e$ y $\Delta e$ son variables lingüísticas de entrada, $\Delta u$ es la variable lingüística de salida, $A$ y $B$ son conjuntos difusos de entrada y $C$ es un conjunto difuso de salida. De las siguientes expresiones basadas en el conocimiento heurístico de los convertidores DC-DC y de la Figura 12 se derivan las 25 reglas mostradas en la Tabla 1.

- Cuando el parámetro controlado está lejos del valor deseado, la variación del ciclo útil debe ser grande, para llevarlo al punto de ajuste rápidamente.

- Cuando el parámetro controlado está cerca del valor deseado, la variación del ciclo útil debe ser pequeño.

- Cuando el parámetro controlado está cerca del valor deseado y se aproxima rápidamente, la variación del ciclo útil debe ser nulo a fin de evitar rebasarlo.

- Cuando se alcanza el valor deseado y el parámetro controlado todavía está cambiando, la variación del ciclo útil debe ser pequeño y con signo opuesto al cambio para prevenir un alejamiento.

- Cuando se alcanza el valor deseado y el parámetro controlado es constante, el cambio del ciclo útil debe ser nulo.

- Cuando el parámetro controlado está por encima del valor deseado, el signo de la variación del ciclo útil debe ser negativo y viceversa en caso contrario.

\section{Mecanismo de inferencia}

El mecanismo de inferencia utilizado en este trabajo comienza obteniendo el factor de peso $w_{i}$ de cada regla mediante la implicación difusa de mínimos de Mamdani de la ecuación (2). 


$$
w_{i}=\mu_{A \cap B}[k]=\min \left\{\mu_{A}(e[k]), \mu_{B}(\Delta e[k])\right\}
$$

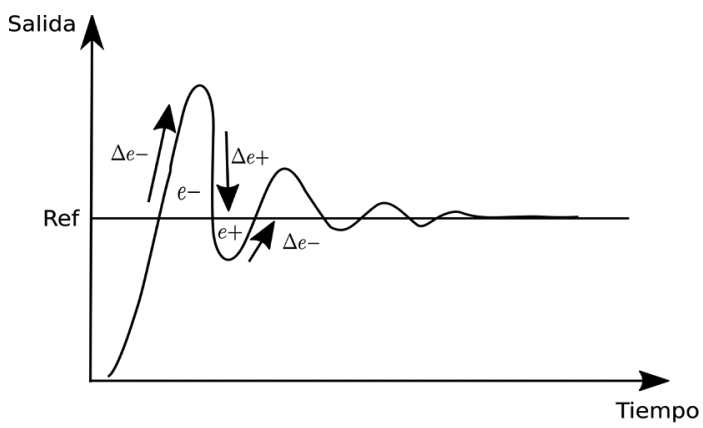

Figura 12. Significado del Error y la Derivada del Error.

Tabla 1. Base de reglas para el controlador de corriente y de voltaje.

\begin{tabular}{|c|c|c|c|c|c|}
\hline \multirow{2}{*}{$\mathbf{e}[\mathbf{k}]$} & \multicolumn{5}{|c|}{$\Delta \mathbf{e}[\mathbf{k}]$} \\
\cline { 2 - 6 } & $\mathbf{N G}$ & $\mathbf{N P}$ & $\mathbf{C E}$ & $\mathbf{P P}$ & $\mathbf{P G}$ \\
\hline NG & NG & NG & NG & NP & CE \\
\hline NP & NG & NG & NP & CE & PP \\
\hline CE & NG & NP & CE & PP & PG \\
\hline PP & NP & CE & PP & PG & PG \\
\hline PG & CE & PP & PG & PG & PG \\
\hline
\end{tabular}

Donde $\mu_{A} e([k]), \mu_{B}(\Delta e[k])$ son los grados de membresía para los valores actuales de $e$ y $\Delta e$ respectivamente.
Entonces, el resultado es aplicado a la función de membresía del consecuente utilizando el método de truncamiento [11], puesto que la parte superior de la función de membresía se recorta el conjunto difuso pierde cierta información, no obstante, este método involucra menos complejidad que otros y genera una superficie de salida agregada fácil de defusificar. Finalmente, las funciones de membresía de todas las reglas previamente recortadas se combinan en un conjunto difuso único utilizando el método de máximos.

\section{Defusificación}

La defusificación fue realizada mediante el método del centroide, el cual encuentra el punto $f_{C G}$ que representa el centro de gravedad del conjunto difuso agregado, obtenido por el mecanismo de inferencia. La representación matemática del proceso está dada por la ecuación (3).

$$
f_{C G}=\frac{\sum_{i=1}^{l} f_{i} * u\left(f_{i}\right)}{\sum_{i=1}^{l} u\left(f_{i}\right)}
$$

Donde $l$ es el número de muestras en el universo de discurso de la variable de salida $\Delta u, f_{i}$ son los valores de las muestras y $u$ el conjunto difuso obtenido al aplicar el procedimiento de inferencia.

El modelo que integra los controladores difusos con el convertidor buck, para implementar el algoritmo de 3 etapas, se muestra en la Figura 13. Los bloques

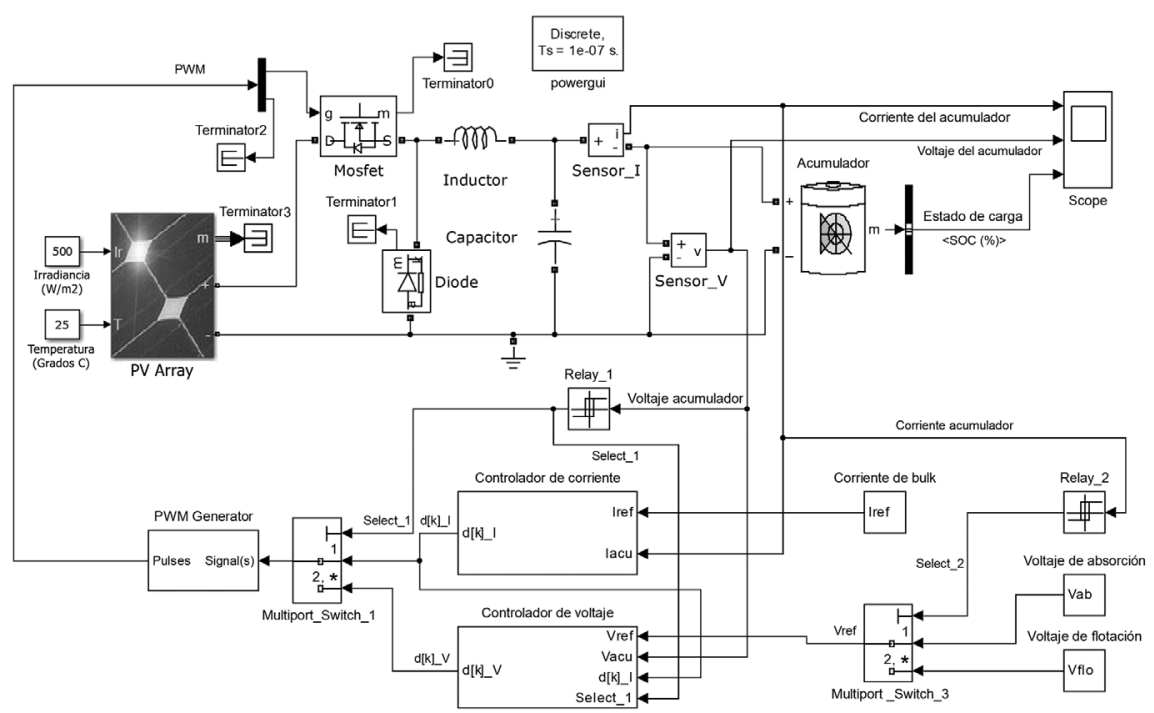

Figura 13. Integración del sistema. 
Relays y Multiport Switches controlan la conmutación entre las etapas del algoritmo, dependiendo del estado de carga del acumulador; Relay_l compara el voltaje de carga del acumulador con el voltaje de absorción y a partir de esto elige mediante Multiport_Switch_l la salida del controlador de corriente o del controlador de voltaje como modulador del módulo PWM, a su vez Relay_2 compara la corriente de carga del acumulador con una corriente de inicio de flotación, permitiendo elegir mediante Multiport_Switch_3 entre el voltaje de absorción y el voltaje de flotación como referencia del controlador de voltaje.

Adviértase la presencia de dos señales de entrada adicionales en el controlador de corriente: $d[k] \_I$ y Select_l, las cuales tiene como función proveer el valor del ciclo útil obtenido por el controlador de corriente para utilizarse como condición inicial del controlador de voltaje, esto es principalmente útil, cuando se conmuta entre las etapas de bulk y de absorción del algoritmo, permitiendo mejorar la respuesta transitoria. Mientras el sistema se encuentra en la etapa de bulk Select_l está deshabilitado, desconectando el integrador en la salida del controlador de voltaje, tal como se muestra en la Figura 14, cuando pasa a la etapa de absorción, Select_l se habilitará conectando el integrador quien tomará como valor inicial el valor de $d[k] \_I$ obtenido en la muestra previa.

En un proceso de carga, los sensores permanentemente obtendrán el voltaje y la corriente en el acumulador; suponiendo que el estado de carga inicial es inferior al $80 \%$, el voltaje muestreado será inferior al voltaje de absorción, por lo que Relay_l habilitará al Multiport_Switch_l para conectar el controlador de corriente como fuente del modulador PWM, éste mantendrá la corriente del acumulador igual a la corriente de bulk tomada como referencia, entrando el sistema en la etapa de bulk. Con estas condiciones, el voltaje de carga comenzará a incrementarse lentamente hasta alcanzar el voltaje de absorción, cuando esto ocurre Relay_l conmuta su estado habilitando al Multiport_Switch_l para conectar el controlador de voltaje, quien mantendrá el voltaje del acumulador igual al voltaje de absorción tomado como referencia, entrando de esta forma a la etapa de absorción del algoritmo. Cuando esto ocurre, la corriente de carga comenzará a disminuirse gradualmente hasta que caiga por debajo de la corriente de inicio de flotación, esta condición conmuta el estado de Relay_2 permitiendo que Multiport_Switch_3 conecte el voltaje de flotación como entrada del controlador de voltaje, entrando a la etapa de flotación.

\section{RESULTADOS}

El diseño fue probado en MATLAB simulando la carga de acumuladores de plomo-ácido con capacidades de $60,80,100$ y $120 \mathrm{Ah}$, utilizando el modelo del panel fotovoltaico y del acumulador incluidos en la librería de Simscape en Simulink, manteniendo las

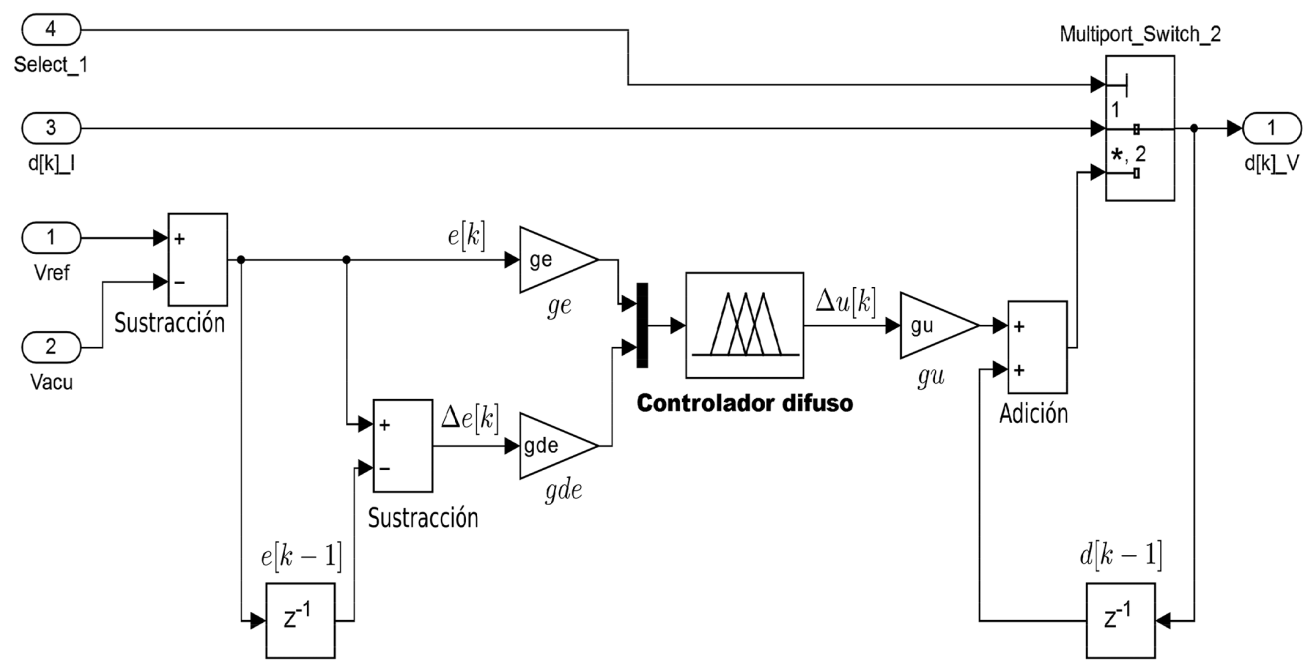

Figura 14. Modificación del controlador de voltaje para mejorar la transición entre etapas del sistema. 
ganancias ya señaladas para ambos controlares. $\mathrm{La}$ Figura 15 muestra el proceso de carga del acumulador de $60 \mathrm{Ah}$, con voltaje de absorción de 14,4 V, voltaje de flotación de $13,8 \mathrm{~V}$, corriente de inicio de flotación de $0,6 \mathrm{~A}$ y un estado de carga inicial del $78 \%$.

La parte de la izquierda de la Figura 15, muestra la conmutación de la etapa de bulk a la etapa de absorción, la cual ocurre en $\mathrm{t}=724,5 \mathrm{~s}$, cuando el estado de carga es aproximadamente igual al $79.916 \%$ para este caso en particular (ver Figura 15c). La Figura 15a muestra como antes de la conmutación el controlador se encuentra en la etapa de bulk, proporcionando una corriente constante e igual a 6 A como se espera (10\% de la capacidad nominal del acumulador) y posteriormente comienza a disminuir gradualmente indicando que ha pasado a la siguiente etapa. La Figura 15b muestra el comportamiento del voltaje de carga, el cual antes de la conmutación se incrementa gradualmente hasta alcanzar 14,4 V (voltaje de absorción) y después de este punto permanece fijo, indicando que el controlador de carga ha entrado a la etapa de absorción. La parte de la derecha de la Figura 15, muestra la conmutación de la etapa de absorción a la etapa de flotación, la cual ocurre en $\mathrm{t}=12,528 \mathrm{~s}$ cuando el estado de carga es aproximadamente igual al 99,933\% (ver Figura 15f). La Figura 15e muestra como después de la conmutación el voltaje de carga permanece fijo en el voltaje de flotación como se espera, a la vez que la corriente de carga disminuye tras un transitorio de 3,5 s (ver Figura 15d).

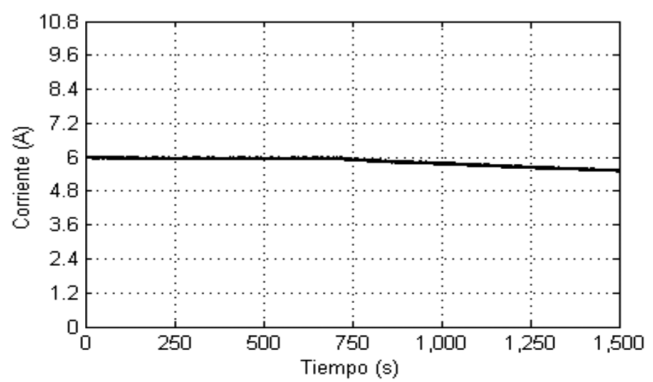

a) Corriente de carga bulk-absorción

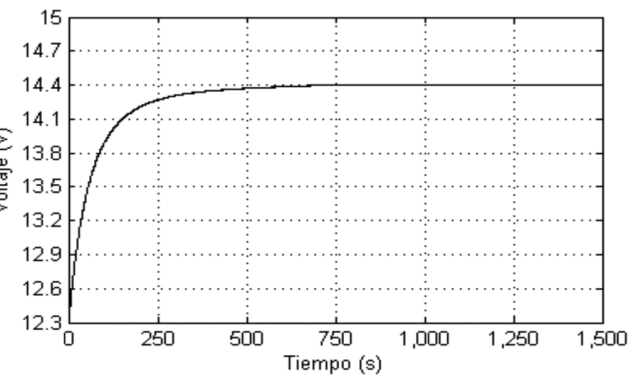

b) Voltaje de carga bulk-absorción

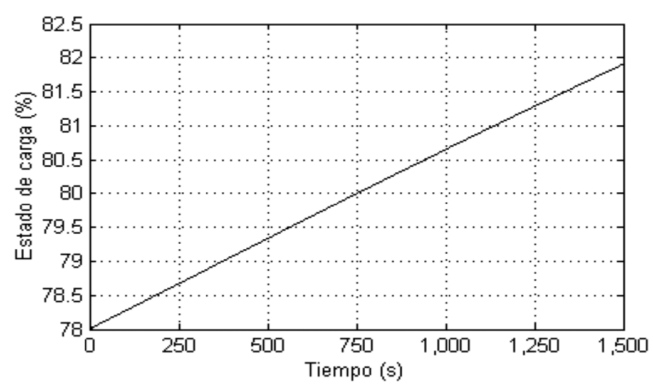

c) Estado de carga bulk-absorción

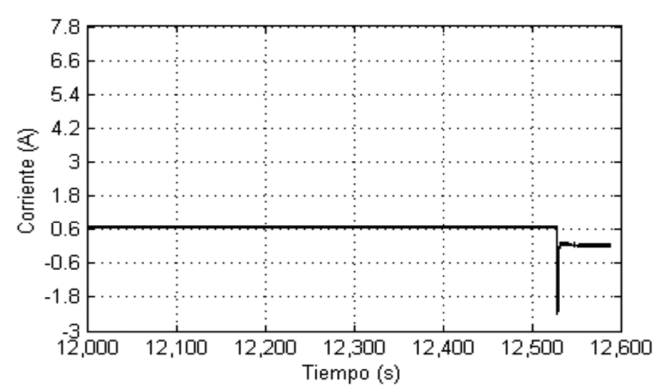

d) Corriente de carga absorción-flotación
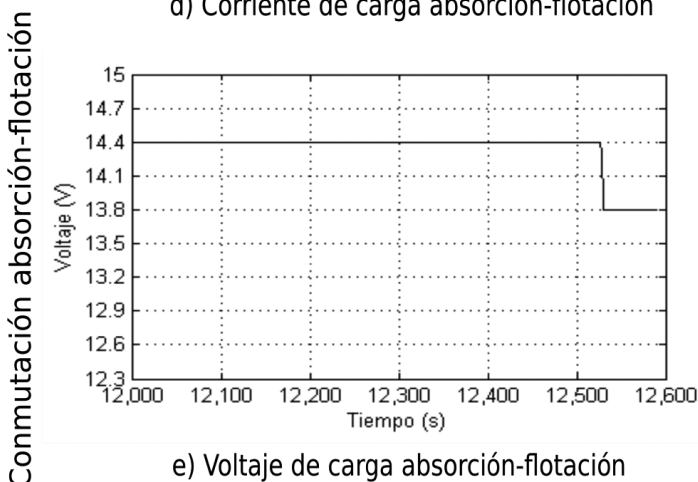

e) Voltaje de carga absorción-flotación

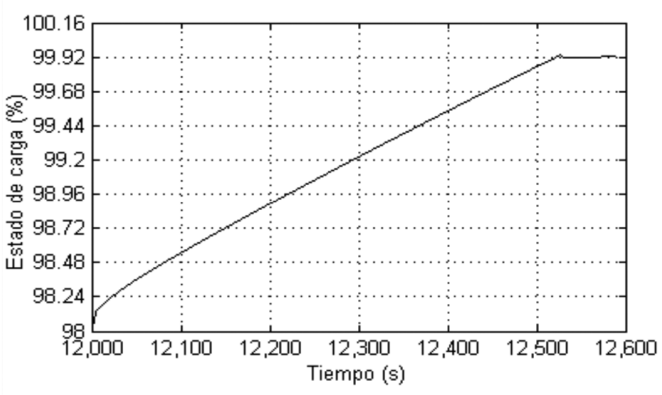

f) Estado de carga absorción-flotación

Figura 15. Simulación del proceso de carga del acumulador de 60 Ah. 
En este punto, es importante resaltar que el convertidor diseñado trabaja en modo continuo, lo que implica una corriente siempre positiva en el inductor, no obstante, en la transición de absorción y flotación se presenta una corriente negativa, lo cual puede evitarse conmutando el convertidor a un modo discontinuo que impida que la corriente circule en sentido contrario, sin embargo, esta mejora queda fuera del alcance de este trabajo. La transición entre los estados de absorción y flotación responde a un cambio súbito (escalón) en el voltaje de carga que pese a ocasionar un sobreimpulso de corriente no presenta oscilaciones (véase Figura 16), lo cual favorece el índice del error dinámico del controlador. Los trabajos relacionados [12] y [13]

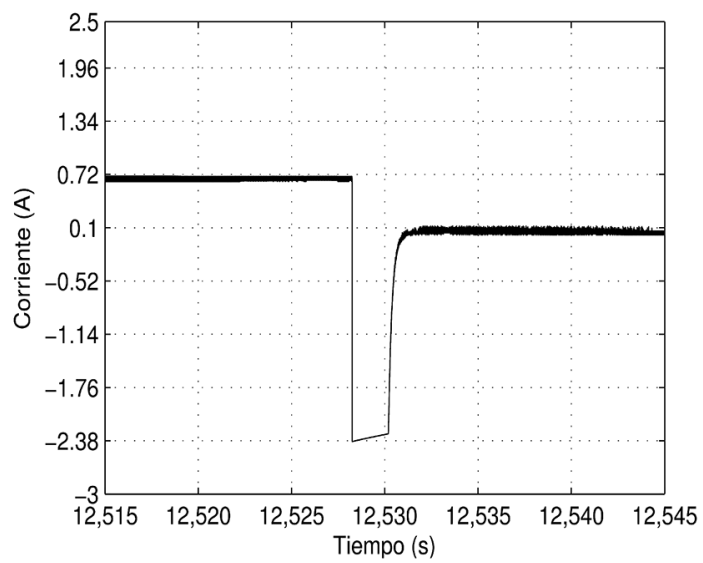

Figura 16. Sobreimpulso de corriente en la transición absorción-flotación. registran tiempos de establecimiento mayores, entre 4 y 30 s respectivamente.

Los tiempos de establecimiento, errores en estado estable y otros parámetros relevantes en la simulación del resto de los acumuladores se resumen en la Tabla 2. En esta tabla puede observarse como en todos los casos el sistema conmuta entre las etapas de bulk y absorción al alcanzar aproximadamente el $79,9 \%$ y el $99,9 \%$ de carga respectivamente, manteniendo en forma precisa los niveles de voltaje y corriente (errores de voltaje y corriente en estado estable inferiores a $0,0002 \mathrm{~V}$ y $0,0015 \mathrm{~A}$, respectivamente), y con tiempos de establecimiento aproximados a 0,01 us en la etapa de absorción y $3 \mathrm{~s}$ en la etapa de flotación.

\section{CONCLUSIONES}

En este trabajo se realizó el diseño y simulación de un controlador de carga basado en un algoritmo de tres etapas: bulk, absorción y flotación, empleando técnicas de control basadas en lógica difusa. El sistema propuesto permitió regular apropiadamente la corriente y el voltaje de carga de acumuladores de plomo-ácido para aplicaciones fotovoltaicas. Los resultados muestran la funcionalidad correcta del diseño, al simular la carga de acumuladores con capacidades de 60 a $120 \mathrm{Ah}$, en todos los casos las transiciones entre etapas del algoritmo son precisas, con tiempos de establecimiento promedio 0,01 us en la etapa de absorción y $3 \mathrm{~s}$ en la etapa de flotación, y errores de corriente y voltaje en estado estable

Tabla 2. Resultados de simulación de carga de acumuladores de 80, 100 y 120 Ah.

\begin{tabular}{|c|c|c|c|}
\hline \multirow{2}{*}{ Descripción } & \multicolumn{3}{|c|}{ Capacidad } \\
\hline & $80 \mathrm{Ah}$ & $10 \mathrm{~A}$ & $120 \mathrm{Ah}$ \\
\hline Corriente en la etapa de bulk & $8 \mathrm{~A}$ & $10 \mathrm{~A}$ & $12 \mathrm{~A}$ \\
\hline Voltaje en la etapa de absorción & $14,4 \mathrm{~V}$ & $14,4 \mathrm{~V}$ & $14,4 \mathrm{~V}$ \\
\hline Voltaje en la etapa de flotación & $13,8 \mathrm{~V}$ & $13,8 \mathrm{~V}$ & $13,8 \mathrm{~V}$ \\
\hline Corriente de inicio de flotación & $0,8 \mathrm{~A}$ & $1 \mathrm{~A}$ & $1,2 \mathrm{~A}$ \\
\hline Punto de conmutación bulk-absorción & $725 \mathrm{~s}$ & $725,5 \mathrm{~s}$ & $726 \mathrm{~s}$ \\
\hline Punto de conmutación absorción-flotación & $12528,5 \mathrm{~s}$ & $12539 \mathrm{~s}$ & $12529,5 \mathrm{~s}$ \\
\hline Estado de carga en la conmutación bulk-absorción & $79,9 \%$ & $79,92 \%$ & $79,93 \%$ \\
\hline Estado de carga en la conmutación absorción-flotación & $99,91 \%$ & $99,94 \%$ & $99,95 \%$ \\
\hline Tiempo de establecimiento bulk-absorción & 0,011 us & 0,010 us & 0,013 us \\
\hline Tiempo de establecimiento absorción-flotación & $2,60 \mathrm{~s}$ & $3,25 \mathrm{~s}$ & $3,87 \mathrm{~s}$ \\
\hline Error de corriente en estado estable & $0,0015 \mathrm{~A}$ & $0,0013 \mathrm{~A}$ & $0,0014 \mathrm{~A}$ \\
\hline Error de voltaje en estado estable & $0,00018 \mathrm{~V}$ & $0,00017 \mathrm{~V}$ & $0,0002 \mathrm{~V}$ \\
\hline
\end{tabular}


inferiores a 0,015 A y $0,0002 \mathrm{~V}$, respectivamente. Estas características garantizan la carga correcta y la protección del acumulador haciéndolo comparable en funcionalidad a diseños similares basados en sistemas de control clásico encontrados en la literatura, pero con un diseño más simple, y apropiado para implementarse directamente en dispositivos económicos como los microcontroladores.

\section{REFERENCIAS}

[1] P. Dinesh, B. Kowshick, P.P. Raghav, R.K. Govindarajan and G.S. Ilango. "Solar Power Based Intelligent Battery Charging System Compatible with Existing Home Inverters". India Educators' Conference (TIIEC), Bangalore, India, 2013.

[2] K. Passino and S. Yurkovich. "Fuzzy Control". Menlo Park, CA: Addison-Wesley. Vol. 20. 1998.

[3] K. Viswanathan, D. Srinivasan and R. Oruganti. "A Universal Fuzzy Controller for a Non-linear Power Electronic Converter". IEEE International Conference on Fuzzy Systems, Honolulu, HI, USA, 2002. DOI: 10.1109/FUZZ.2002.1004957.

[4] P. Mattavelli, L. Rossetto, G. Spiazzi and P. Tenti. "General-purpose fuzzy controller for DC-DC converters". IEEE Transactions on Power Electronics. Vol. $12 \mathrm{~N}^{\mathrm{o}}$ 1. pp. 79-86, 1997. DOI: $10.1109 / 63.554172$.

[5] F. Wang and C.Q. Lee. "Comparison of Fuzzy Logic and Current-Mode Control Techniques in Buck, Boost and Buck/Boost Converters". Power Annual Power Electronics Specialists Conference. Atlanta, GA, USA, 1995. DOI: 10.1109/PESC.1995.474949.
[6] S. Armstrong, M.E. Glavin and W. Hurley. "Comparison of Battery Charging Algorithms for Stand Alone Photovoltaic Systems". Power Electronics Specialists Conference. Rhodes, Greece, 2008. DOI: 10.1109/ PESC.2008.4592143

[7] D. Hart. "Electrónica de Potencia". Madrid, España: Prentice Hall. 2001.

[8] M. Shyama and P. Swaminathan. "Digital Linear and Nonlinear Controllers for Buck Converters". International Journal of Soft Computing and Engineering. Vol. $2 \mathrm{~N}^{\mathrm{o}} 1$, pp. 336-342. 2012.

[9] L. Guo. "Design and Implementation of Digital Controllers for Buck and Boost Converters using Linear and Nonlinear Control Methods". Auburn, Alabama. 2006.

[10] L.-X. Wang. "A Course in Fuzzy Systems and Control". London, Inglaterra: Prentice Hall. 1997.

[11] I. Iancu. "A Mamdani Type Fuzzy Logic Controller". Prof. Elmer Dadios (ed.). 2012.

[12] R. Swathika, R.G. Ram, V. Kalaichelvi and R. Karthikeyan. "Application of fuzzy logic for charging control of lead-acid battery in stand-alone solar photovoltaic system". International Conference on Green Computing, Communication and Conservation of Energy. Chennai, India. 2013. DOI: 10.1109/ICGCE.2013.6823464.

[13] V.J. Greeshma and S. Revathy. "Battery charging control using fuzzy logic based controller in a photovoltaic system". International Advanced Research Journal in Science, Engineering and Technology. Vol. $3 \mathrm{~N}^{\mathrm{o}}$ 3, pp. 114-117. 2016. 